SHS Web of Conferences 21, 03003 (2015)

DOI: $10.1051 /$ shsconf $/ 20152103003$

(C) Owned by the authors, published by EDP Sciences, 2015

\title{
Les rencontres des enfants avec le vivant à l'école maternelle
}

\author{
Frédéric Charles ${ }^{\mathrm{a}}$ \\ Laboratoire S2HEP, EA 4148, Université Claude Bernard Lyon 1 et École Normale Supérieure de \\ Lyon, France
}

\begin{abstract}
Résumé. Fréquentée par la quasi-totalité des enfants âgés de 3 à 6 ans, l'école maternelle française doit permettre une entrée réussie dans la culture scientifique, notamment une exploration du milieu naturel. Les enseignants de maternelle organisent des rencontres entre les enfants et le vivant. Ces rencontres initient le curriculum d'éducation biologique. Dans une perspective curriculaire, une double investigation des pratiques des enseignants a été menée par un questionnaire $(\mathrm{N}=92)$ et par des carnets de bord et des entretiens $(\mathrm{N}=12)$. L'analyse des données permet de reconstituer les rencontres des élèves avec le vivant. Les résultats montrent qu'elles sont nombreuses, organisées autour d'observations d'élevages et de plantations. L'analyse des pratiques permet de repérer que les enseignants visent non seulement l'enrichissement du capital expérientiel des enfants, mais aussi l'acquisition de premières connaissances plus formalisées sur le vivant. Les pratiques sont également caractérisées par leur caractère improvisé, ce qui peut entrainer parfois des incohérences au plan épistémologique, notamment dans la programmation et la mise en œuvre au fil du temps de ces rencontres entre les élèves et le vivant.
\end{abstract}

\begin{abstract}
Attented by nearly all children aged 3 to 6 years, French nursery school must allow a successful entry in the scientific culture, including an exploration of the natural environment. Nursery school teachers organize encounters between children and the living. These encounters initiate the biological education curriculum. In a curricular perspective, a double investigation of teachers practices was conducted by a questionnaire $(\mathrm{N}=92)$, and logbooks and interviews $(\mathrm{N}=12)$. Data analysis helps to replenish encounters between pupils and the living. The results show that these meetings are frequent, organized around observations from farms and plantations. The analysis of practices identify that teachers aimed not only the enrichment of children' experiential capital but also the acquisition of more formalized knowledge about live and living. The practices are also characterized by their improvised nature, which can sometimes lead to epistemological inconsistencies, especially in the planning and implementation of these encounters between pupils and the living.
\end{abstract}

Auteur de correspondance : frederic.charles@univ-lyon1.fr

This is an Open Access article distributed under the terms of the Creative Commons Attribution License 4.0, which permits unrestricted use, distribution, and reproduction in any medium, provided the original work is properly cited. 


\section{Introduction}

Cet article présente une analyse de données issues d'une recherche doctorale (Charles, 2012) 1 pensée en fonction de la problématique du colloque «Vie et Vivant », contribuant au volet 3 relatif au milieu scolaire. Cette analyse vise à reconstituer les rencontres avec le vivant offertes aux enfants de l'école maternelle française par leurs professeurs des écoles. Ces rencontres constituent les prémices du curriculum d'éducation biologique de ces jeunes enfants âgés de 2 à 6 ans.

Dans une perspective de didactique curriculaire (Bisault, 2011 ; Lebaume, 2003) 2 3, une double investigation des pratiques des enseignants de maternelle a été réalisée par un questionnaire $(\mathrm{N}=92)$ et par un suivi de professeurs avec des carnets de bord complétés d'entretiens semi-directifs $(\mathrm{N}=12)$. La description et l'analyse des pratiques enseignantes permettent de comprendre les rencontres qu'ils organisent entre leurs élèves et le vivant.

Après avoir présenté le contexte et les enjeux socio-éducatifs de la recherche, le cadre de référence légitimant l'investigation des pratiques enseignantes sera exposé. Les résultats focaliseront deux points : tout d'abord, les activités proposées aux enfants, ensuite les visées que les enseignants associent à ces activités. La caractérisation des rencontres des enfants avec le vivant permet finalement de discuter les incohérences épistémologiques dans les pratiques, notamment dans la programmation et la mise en œuvre au fil du temps de ces activités biologiques.

\section{Contexte et enjeux socio-éducatifs}

L'école maternelle française occupe une place particulière au sein du paysage des structures d'accueil de la petite enfance. En premier lieu, depuis les années 1950-1960, sa fréquentation s'est amplifiée fortement. C'est ce mouvement qu'Antoine Prost (1981) 4 qualifie d' "école maternelle pour tous ». $\mathrm{Au}$ delà de son importante fréquentation au plan quantitatif ${ }^{1}$ - la quasi-totalité des enfants de 3 à 6 ans y est scolarisée -, l'école maternelle est reconnue par tous les milieux sociaux-professionnels, et en particulier les milieux favorisés (Plaisance, 1986) 5.

En second lieu, la maternelle se distingue par son orientation fondamentalement éducative prise dans les années 1960 (Prost, 1981) 4. Des instructions officielles prescrivent le travail des professeurs des écoles affectés en maternelle. Dans les programmes en vigueur à la rentrée 2015 (Ministère de l'Éducation Nationale, 2015) 6, tout comme les précédents (Ministère de l'Éducation Nationale, 2008) 7, le domaine d'apprentissage étiqueté «Explorer le monde du vivant, des objets et de la matière » renvoie à une première éducation scientifique et technologique. Pour les concepts de «vie » et de «vivant», ce texte recommande une exploration active du monde vivant par les enfants, pour qu'ils commencent à identifier certaines caractéristiques du monde vivant et à comprendre certaines distinctions du vivant et du non-vivant. L'absence du terme «vie» dans ces prescriptions et la forte occurrence du terme « vivant » amènent à privilégier dans ce texte l'usage du mot «vivant».

L'enjeu socio-éducatif est donc fort, car les enseignants de l'école maternelle doivent assurer ce que Christian Orange et Élisabeth Plé (2000) 8 appellent une «entrée réussie dans la culture scientifique et technologique» pour les petits français fréquentant cette école. Cette première formation à l'exploration du vivant doit offrir la possibilité d'une capitalisation expérientielle contribuant ainsi à la constitution d'un premier référent empirique (Coquidé \& Lebeaume, 2003) 9. Maryline Coquidé (1998) 10 précise le sens de ces premières expériences en situant l'école maternelle comme lieu de familiarisation pratique. Il s'agit alors d'expériences-actions et d' «expérienciation », où le terme «expérience», pris dans son acception psychologique (au sens du terme anglais

\footnotetext{
${ }^{1}$ En effet, l'école maternelle est caractérisée par sa grande fréquentation : elle accueille en effet la quasi-totalité des jeunes français âgés de deux à six ans : environ un quart $(23,5 \%)$ des enfants de deux ans, $95 \%$ de ceux de trois ans et $98 \%$ - voire plus - des enfants de quatre et cinq ans (Insee, 2009).
} 
experience), valorise l'expérience propre de l'enfant. Durant la scolarité à l'école maternelle, ces expériences de vie initient le parcours éducatif, ou curriculum d'éducation biologique, constitué par l'ensemble des rencontres des enfants avec le vivant.

\section{Problématique}

Le curriculum d'éducation biologique dépend des mises en œuvres des enseignants. La recherche présentée vise la description et l'analyse des pratiques des professeurs des écoles qui organisent les rencontres entre leurs élèves et le vivant.

Si les recherches en sciences de l'éducation préoccupées par les pratiques professionnelles des enseignants focalisent souvent leur travail quotidien en privilégiant les dimensions écologiques et pédagogiques, les travaux de didactique des sciences restent restreints. Une recension bibliographique montre que les recherches investiguant les pratiques enseignantes ne sont pas les plus nombreuses dans le champ des didactiques des sciences et des techniques. De façon encore plus fragrante, celles consacrées à la première éducation scientifique et technologique à l'école maternelle sont encore limitées. La thèse de Lasson (2004) 11 traite en partie cette question pour la découverte du monde des objets à l'école maternelle. Celle de Catherine Ledrapier (2007) 12 examine la possibilité dès l'école maternelle d'une éducation scientifique par une exploration active du monde de la matière en convoquant un cadre théorique relatif à la didactique et à la psychologie des apprentissages.

Plus récemment, en $2014^{2}$, un symposium " l'éducation scientifique et technique à l'école maternelle : des interrogations didactiques» tentait de faire le point sur des connaissances sur les enjeux éducatifs, les perspectives didactiques et les approches de recherche relatifs à cette éducation. Ce sont essentiellement des perspectives curriculaires (Bisault, 2014) 13, comparatistes (Coquidé, 2014) 14 ou bien de professionnalité (Charles, 2014) 15 que les chercheurs ont mobilisé. Plus précisément, les questions concernent les spécificités et les enjeux éducatifs de ce segment scolaire, les caractéristiques du développement du jeune élève de maternelle, les perspectives expérientielles mises en œuvre, le rôle des enseignants dans l'élaboration des activités, leur organisation spatiotemporelle, et leurs connexités, en particulier avec des activités à visée langagière et/ou artistique.

De façon plus large, le panorama des recherches sur l'école maternelle montre une centration sur les processus d'enseignement-apprentissage, en lien avec les didactiques disciplinaires, ce qui est incompatible avec les spécificités de ce segment scolaire mises en avant par Florin et Crammer (2009) 16. En effet, les disciplines sont encore absentes à l'école maternelle et les pratiques enseignantes sont souvent marquées par des mises en œuvre de séance dont les visées sont multiples et entremêlées. Cet article contribue donc à combler le déficit de travaux de recherche relatifs à la première éducation en biologie à l'école maternelle, privilégiant l'approche didactique, la prise en compte des contenus, de leurs spécificités et de leur organisation.

L'objet de la recherche, les rencontres des enfants avec le vivant, initiant le curriculum d'éducation biologique, oblige à adopter une approche globale de l'ensemble de la structure du curriculum. Connaître et comprendre cet ensemble de rencontres suppose une temporalité longue, et une inscription de cette étude dans la perspective des travaux de didactique curriculaire, proposée par Lebeaume (2000) 3 et développée à l'école primaire par Bisault (2011) 2.

\section{Méthodologie}

Pour mettre au jour les rencontres avec le vivant proposés aux enfants de l'école maternelle, les choix méthodologiques ont privilégié un recueil de données centré sur les pratiques des enseignants, considérés comme les organisateurs de ces rencontres. Deux investigations complémentaires ont été retenues : ${ }^{2}$ Ce symposium a été organisé lors des $8^{\text {èmes }}$ rencontres scientifiques de l'ARDIST (l'Association pour la
Recherche en Didactique des Sciences et des Technologies) qui ont eu lieu à Marseille du 12 au 14 mars 2014 . 
- La première est une approche qualitative reposant sur l'analyse de données empiriques, constituées par des carnets de bord tenus par 12 enseignants durant une année scolaire ${ }^{3}$. Cette grande échelle de temporalité permet de préciser les rencontres proposées aux enfants avec le vivant. Les carnets de bord ont été renseignés par des enseignants volontaires pour contribuer à la recherche. Lors de la première rencontre, il était précisé l'engagement long et régulier qui était demandé, notamment de consigner, dans le carnet, les éléments relatifs à la pratique d'éducation biologique. Les écrits, entre récit et description de la réalité perçue, sont généralement rédigés à la première personne et possèdent les caractéristiques d'une écriture réflexive et professionnalisante selon la typologie de Cros (2003) 17. Trois entretiens semidirectifs ont pris appui sur ces traces de l'activité enseignante (Vinatier, 2009) 18 selon une fréquence moyenne d'un entretien par trimestre avec chacun des enseignants. Ces entretiens ont permis l'exploration des parties lacunaires des carnets de bord. La transcription du contenu des carnets de bord et des entretiens a constitué un premier ensemble de données permettant la restitution des pratiques enseignantes.

- La seconde investigation, plus quantitative, correspond à un questionnaire. L'analyse des pratiques déclarées par 92 enseignants permet d'affiner et d'étayer les tendances repérées par la première méthode. Les destinataires de ce questionnaire sont des participants au $83^{\text {ème }}$ congrès de l'AGEEM ${ }^{4}$. Lors de ce congrès, 300 questionnaires ont été distribués librement sur le site, et un tiers retourné. Ce sont tout d'abord les questions relatives aux contenus, activités et visées des rencontres avec le vivant qui sont analysées pour cette contribution. Ensuite, ce sont les réponses aux questions proposant des scenarii didactiques qui sont examinées. Dans ces propositions, il s'agit d'explorer les priorités accordées dans ces rencontres à propos de contenus susceptibles d'être pris en charge à l'école maternelle : les enseignants ont à hiérarchiser des objectifs dans une liste proposée.

Dans la perspective curriculaire retenue, l'analyse du contenu des données empiriques issues des carnets et des entretiens utilise une grille établie selon le schéma d'analyse de Lebeaume (2000) 3. Elle permet de caractériser les rencontres entre les enfants et le vivant en précisant notamment les contenus pris en charge par les enseignants, les tâches associées des élèves et leurs visées éducatives. Cette grille permet de reconstruire le curriculum d'éducation biologique des enfants à l'école maternelle.

Les données recueillies par questionnaire sont traitées de manière quantitative et qualitative, par une reprise de la grille des carnets de bord et entretiens et un traitement statistique.

\section{Résultats}

\subsection{Que rencontrent les enfants à l'école maternelle ?}

Au plan quantitatif, les résultats montrent que les enseignants organisent de rencontres régulières et nombreuses entre les enfants et le vivant :

- En effet, 11 des 12 enseignants suivis avec les carnets de bord mettent en œuvre des activités.

- Seul 1 enseignant sur les 92 questionnés déclare ne pas prendre en charge les activités de découverte du monde du vivant.

\footnotetext{
${ }^{3}$ Ces enseignants ont été sollicités au cours de stages de formation ou après consultation d'inspecteurs de circonscription de trois départements de la région Champagne-Ardenne. Afin de soutenir l'engagement dans une recherche longue, des contacts réguliers ont permis de répondre aux questions tout en valorisant l'implication des enseignants.

${ }^{4}$ AGEEM $=$ Association Générale des Enseignants des Écoles et classes Maternelles publiques. Le $83^{\text {ème }}$ congrès, réunissant un millier d'enseignants, s'est tenu à Annecy du $1^{\mathrm{er}}$ au 3 juillet 2010.
} 
Par comparaison avec d'autres données sur les mondes des objets et la matière (tableau 1), l'analyse du contenu des réponses prouve de façon forte que le vivant est la partie privilégiée par les enseignants, certes quantitativement, mais aussi qualitativement : les activités décrites sont en effet plus variées que celles relatives aux objets et à la matière.

Les rencontres des enfants avec le monde du vivant sont donc nombreuses alors que celles avec le monde de la technique restent rares. Ces résultats convergent non seulement avec ceux de Lasson (2004) 11 qui montre que le monde des objets est très réduit à l'école maternelle, mais aussi avec ceux de Baillat $(2001$; 2003) 19, 20 concernant les classes de fin d'école primaire, qui met en évidence la priorité accordée à la biologie et la place mineure accordée par les enseignants aux activités technologiques.

Tableau 1. Les mondes rencontrés par les enfants de maternelle.

\begin{tabular}{|c|c|}
\hline $\begin{array}{c}\text { Monde pris } \\
\text { en charge }\end{array}$ & $\begin{array}{c}\text { Nombre d'enseignants } \\
\text { (12 suivis) }\end{array}$ \\
\hline Le vivant & 11 \\
\hline (La matière & 7 \\
\hline Les objets & 2 \\
\hline
\end{tabular}

Les pratiques enseignantes sont marquées par :

- Une presque omniprésence du monde végétal. En effet, $88 \%$ des enseignants déclarent permettre des rencontres entre les enfants et les végétaux. Les activités mises en œuvre sont des activités de plantations et de jardinage. C'est en fait le jardin qui est très souvent un lieu de rencontre fort entre les élèves et les végétaux : $42 \%$ des enseignants font en effet un jardin dans leur école, permettant souvent des plantations de bulbes (l'Amaryllis est celui le plus cité) et/ou des semis de graines (de plantes vivaces, aromatiques, etc.).

- Une forte présence du monde animal dans les écoles maternelles. 62\% des enseignants déclarent en effet mettre en œuvre des élevages dans leurs classes, favorisant ainsi des rencontres entre les enfants et les animaux. L'analyse du contenu des réponses permet de préciser les «animaux-vedettes» de la maternelle : l'escargot, les chenilles/papillons, les poissons, les phasmes, les petits mammifères et les coccinelles sont les animaux les plus fréquemment cités par les enseignants et que les enfants rencontrent.

- $\quad$ En plus de ces êtres vivants, végétaux et animaux, les enseignants organisent des rencontres avec un autre représentant du monde vivant, l'Homme. En effet, les enfants sont amenés à explorer leur corps et son fonctionnement, notamment grâce à l'étude du schéma corporel (évoquée par plus d'un quart des questionnés), à une éducation sensorielle et à la santé (15\% des réponses au questionnaire) et à une approche de la croissance (10\% des questionnés déclarent aborder ce phénomène).

\subsection{Quelles activités sont menées lors de ces rencontres ?}

Les réponses des questionnés montrent que pour 35\% d'entre eux, les rencontres avec le vivant s'organisent autour d'activités d'observation. Cette forte place de l'observation prouve que les enseignants associent volontiers cette pratique aux activités «reines » que sont les élevages et les plantations/jardins. Presque $25 \%$ des réponses présentent une cooccurrence du type «élevages, plantations et observations » qui semble constituer une trilogie représentative des pratiques. La pratique d'Amélie, une enseignante de grande section suivie par carnet de bord (extrait 1), témoigne de l'association élevage/observation. En effet, dans les élevages de chenilles et de phasmes qu'elle met en place dans sa classe, elle met en œuvre de réelles activités d'observation (Guichard, 1988) 21 
liées à un questionnement scientifique légitimant l'observation et les dessins demandés aux enfants, favorisant non seulement l'apprentissage de cet exercice graphique, mais aussi l'observation ellemême.

Un autre type d'activité déclaré mis en œuvre par un quart des enseignants tourne autour des visites et des sorties, qui permettent de multiples rencontres entre les élèves et le monde du vivant. Ces visites et sorties, que les enseignants qualifient de «pédagogiques » ont lieu le plus souvent dans la cour de l'école, les bois, les parcs, les mares, les lacs ou les rivages marins. Ces études des milieux proches des enfants permettent aux enseignants de leur faire découvrir «la faune», la «flore» ou encore «les petites bêtes ».

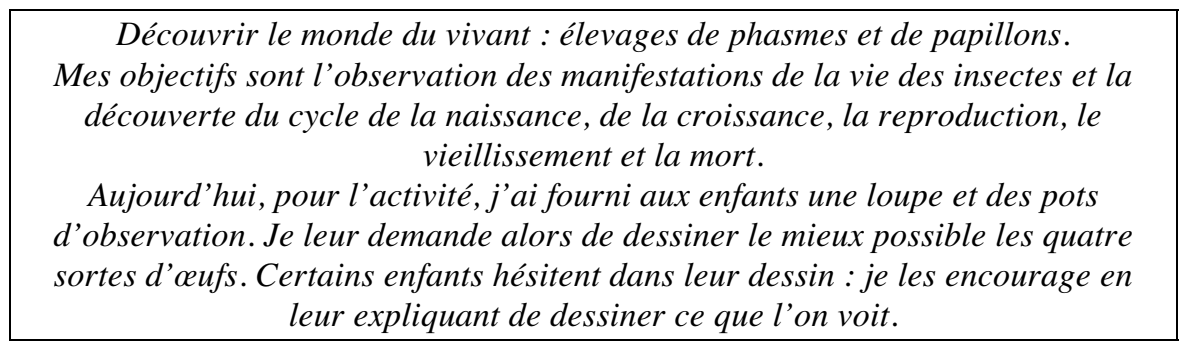

Extrait 1. Carnet de bord de l'enseignante Amélie.

\subsection{Qu'est-ce qui est visé lors de ces rencontres?}

Lors des activités décrites précédemment, les enseignants visent une première familiarisation pratique des enfants avec le vivant. L'analyse des données montre qu'au delà de cette première familiarisation, ils travaillent aussi des «contenus-conceptualisations» lorsqu'ils organisent les rencontres précédemment décrites. Cette expression, en référence au concept de registre de conceptualisation proposé par Michel Develay (1992) 22, désigne un premier palier de conceptualisation. Parmi ces contenus-conceptualisations, c'est la croissance (des végétaux et des élèves eux-mêmes) qui est évoquée majoritairement, puis l'alimentation (des enfants et des animaux élevés). Le concept de cycle de vie est également beaucoup travaillé lors des rencontres avec les animaux et les végétaux : les enseignants déclarent en effet aborder la naissance, la croissance, la reproduction et la mort. L'extrait 2 illustre ces aspects lors d'un élevage de têtards réalisé dans une classe de moyenne/grande section.

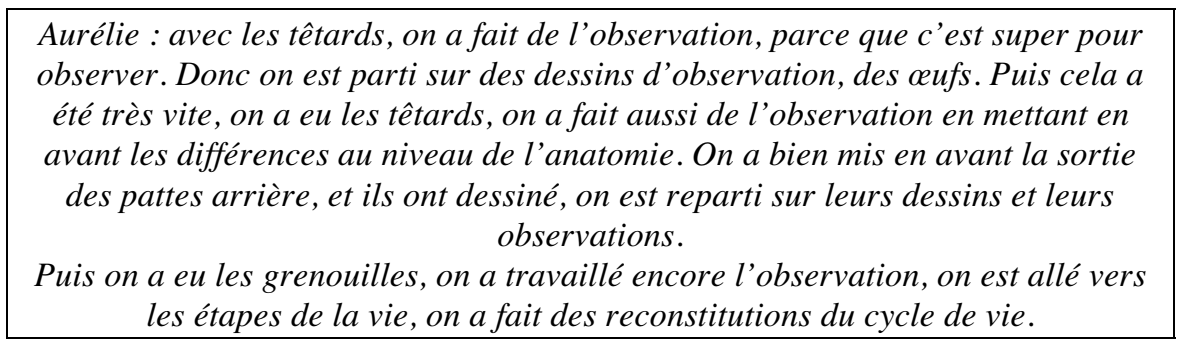

Extrait 2. Troisième entretien avec l'enseignante Aurélie.

Aurélie vise à la fois des capacités d'observation chez les enfants, mais aussi un premier niveau d'élaboration intellectuelle à propos du développement indirect. L'enseignante Amélie (extrait 3) précise également cette visée lors d'élevages de phasmes et de chenilles/papillons qui lui permettent de faire prendre conscience chez les enfants de certaines différences entre développement direct et développement indirect. 
Chercheur : qu'est-ce qui était important pour toi avec les élevages?

Amélie : et bien donc, ce qui était important, c'était que les enfants voient l'évolution du stade " œuf » à l'animal, l'insecte, le phasme et le papillon adulte. Je voulais qu'ils puissent constater les différentes étapes du cycle de la vie. Les différentes étapes d'une naissance et puis de la croissance.

Extrait 3. Deuxième entretien avec l'enseignante Amélie.

L'exploration des priorités accordées lors de ces rencontres par les scenarii didactiques du questionnaire permet de fournir des données qui convergent avec les suivis par carnets de bord et entretiens. En effet, l'analyse des réponses aux divers scenarii montre que les enseignants visent prioritairement des concepts lors des rencontres des enfants avec le vivant alors qu'ils privilégient l'enrichissement de l'expérience ou le langage lors des moments de découverte de la matière ou des objets. L'extrait 4 présente le scenario sur les « Activités de jardinage en moyenne section».

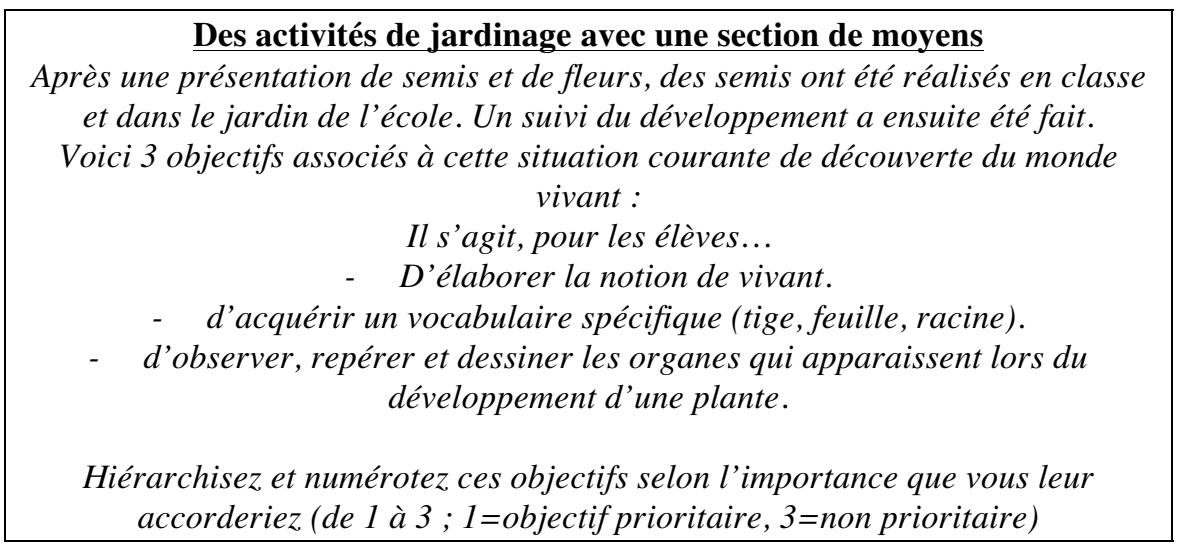

Extrait 4. Un scenario didactique en lien avec le monde vivant (présent dans le questionnaire).

Ce scenario, correspondant à des activités de jardinage en moyenne section, proposait un premier objectif centré sur la connaissance (un premier niveau de conceptualisation du vivant), le deuxième sur un choix langagier lié à l'apprentissage de vocabulaire et le troisième à des savoir-faire rattachés à l'éducation scientifique.

Les résultats à cette question sont présentés sur la figure 1 . 


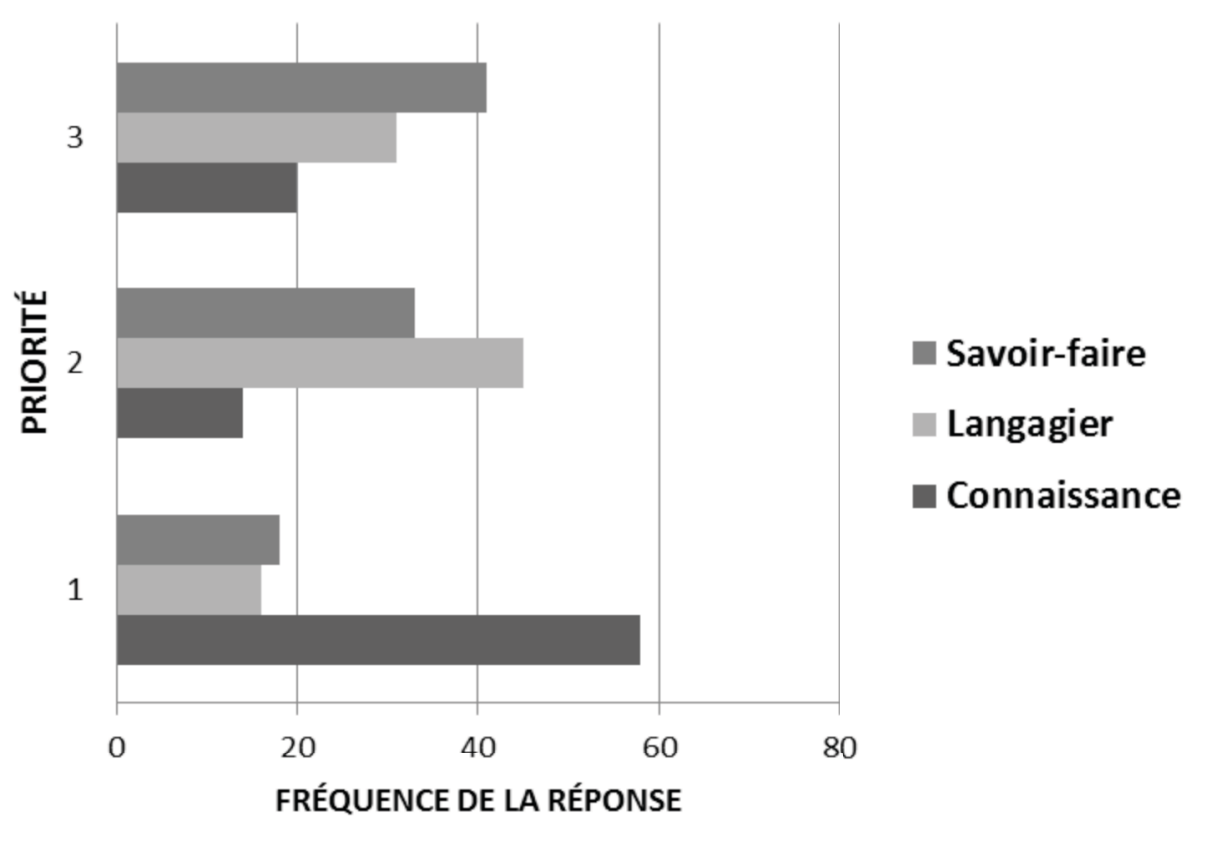

Figure 1. Répartition des choix des questionnés à propos du scenario didactique « Des activités de jardinage avec une section de moyens $»$.

Les résultats, examinés longitudinalement ou transversalement sont cohérents. Ils montrent que l'ordre de priorité le plus récurrent part de la visée de connaissance, puis langagier et enfin savoirfaire. Les enseignants (58\% d'entre eux) accordent donc leur priorité à l'élaboration première de la notion de vivant.

L'extrait 5 présente la proposition de scenario portant sur « Flotter et couler en moyenne et grande sections ».

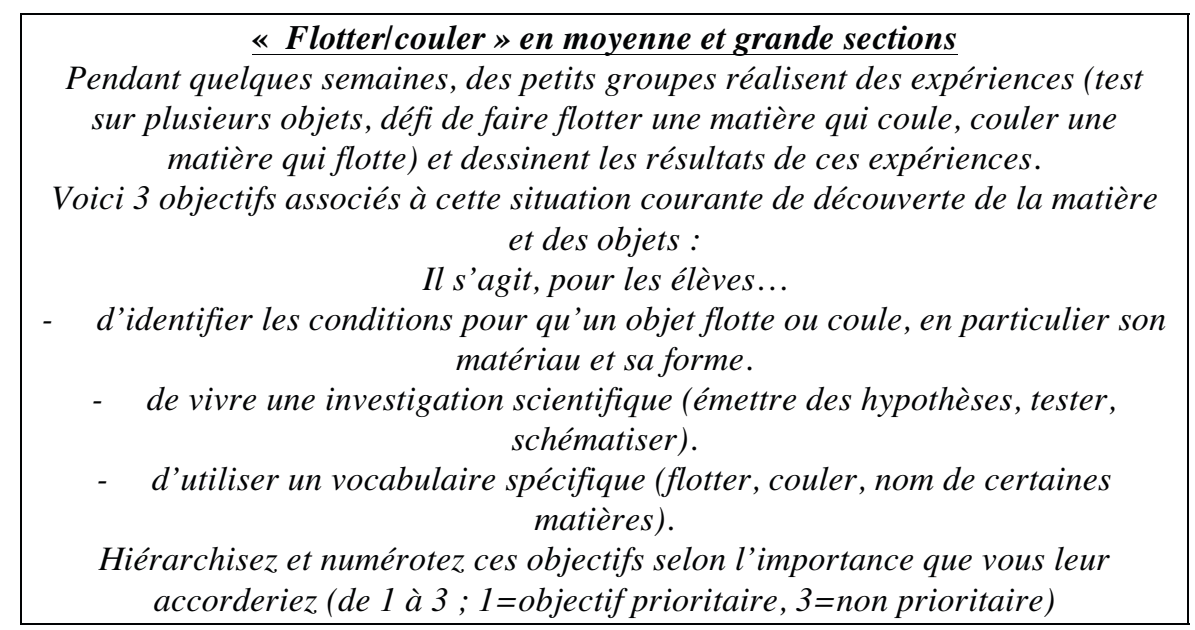

Extrait 5. Un scenario didactique en lien avec le monde de la matière (présent dans le questionnaire).

Ce scenario didactique concernait des activités autour des phénomènes de flottaison avec des élèves de moyenne et grande sections. Le premier objectif proposé visait des connaissances, le 
deuxième se centrait sur la démarche (et donc des savoir-faire associés), le troisième sur l'acquisition de vocabulaire. Les résultats à cette question sont présentés sur la figure 2.

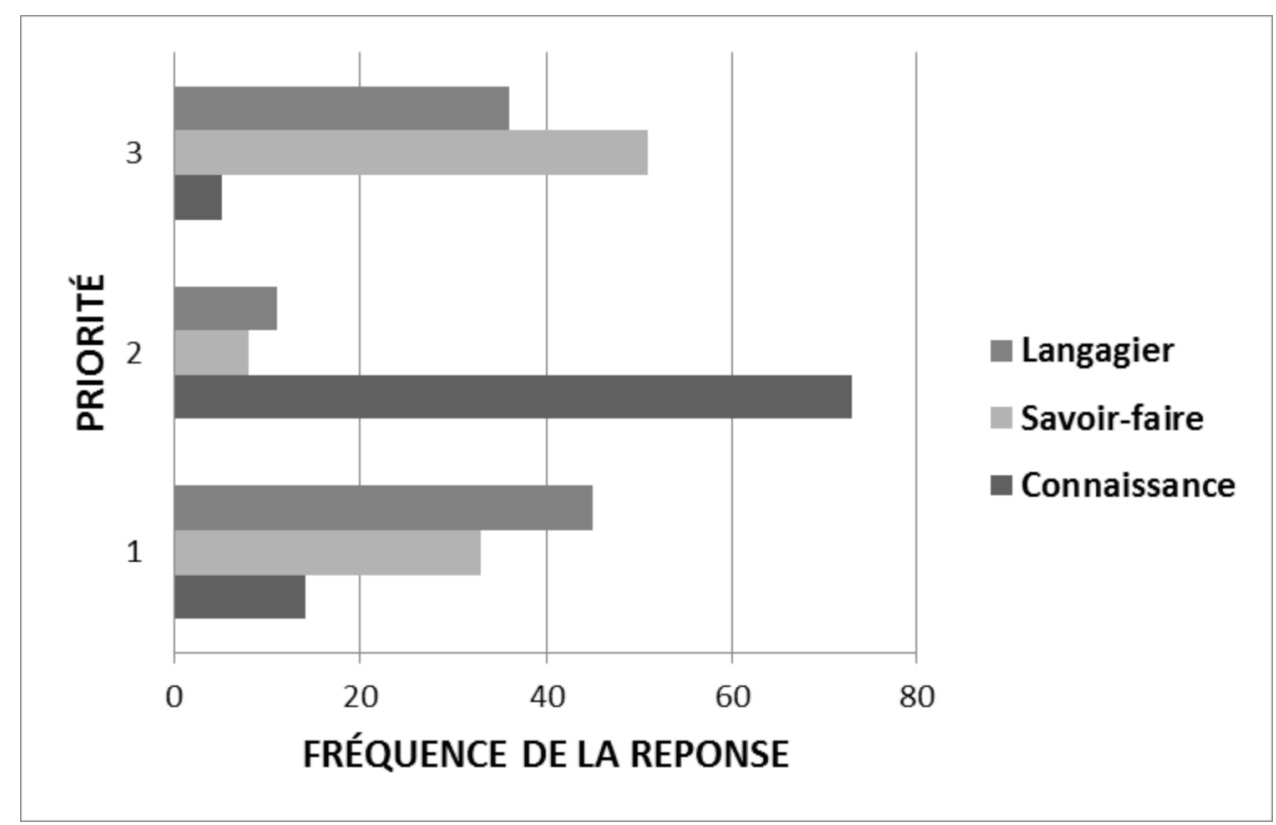

Figure 2. Répartition des choix des questionnés à propos du scenario didactique « Flotter/Couler en moyenne et grande sections ».

Les résultats n'ont pas totalement la même logique selon l'angle de lecture du graphique. Au $1^{\text {er }}$ rang, le langage, puis le savoir-faire et enfin la connaissance sont visés. Au dernier rang, le savoirfaire, le langage et pour finir la connaissance ne sont pas une priorité pour ces enseignants. Malgré une certaine dispersion des réponses dans ce scenario, deux éléments sont à noter : le premier est que la visée de langage est privilégiée ; le second est que la connaissance est jugée non prioritaire, choisie par seulement $13 \%$ des interrogés sur ce scenario lié au monde des objets et de la matière.

Ainsi, l'ensemble des données montre que les enseignants, lorsqu'ils organisent des rencontres entre les enfants et le vivant, visent non seulement une capitalisation expérientielle, mais aussi la construction de premières élaborations intellectuelles.

\section{Discussion et conclusion}

Dans cette recherche dont la visée était de caractériser les rencontres des élèves des écoles maternelles avec le vivant, les résultats montrent clairement que les enseignants organisent des rencontres nombreuses non seulement quantitativement, mais aussi qualitativement : le monde du vivant est bien présent dans les écoles maternelles.

Une enquête historique menée à partir de l'analyse de prescriptions primaires et secondaires (1881-2012) permet de démontrer l'enracinement historique profond des pratiques contemporaines mises au jour (Charles, 2013) 23 et de débusquer l'existence de «normes professionnelles spécifiques» (Prairat, 2012) 24 déterminant des façons de penser et des manières d'agir des enseignants de maternelle. Parmi ces normes, la prédominance de la dimension du tissage dans le modèle des gestes professionnels des enseignants (Bucheton et Soulé, 2009) 25 doit être soulignée, donnant naissance à un réseau de séances articulées entre elles de manière cohérente.

Au delà de la préparation et de l'organisation pédagogique de ces nombreuses rencontres, les pratiques enseignantes sont caractérisées par la présence de l'improvisation. C'est l'analyse des 
réponses à une question qui permet d'approcher ce caractère (extrait 6). Cette question explorait le pilotage de la planification au cours du temps des rencontres entre les enfants et le monde du vivant. Trois assertions étaient à hiérarchiser. L'une d'elle proposait une planification fixée à l'avance, une autre une planification relativement fixe, mais malléable en fonction d'opportunités et la dernière renvoyait à une planification plus souple et fortement pilotée par les remarques des enfants.

Planifier les rencontres avec la vie et le vivant
Pour cette question, numérotez par ordre croissant les propositions depuis celle
où vous êtes le plus en accord (=1) à celle où vous êtes le moins en accord.
À propos de la planification des activités de découverte du monde du vivant :
- En début d'année, vous avez planifié ces activités selon une progression
précise.
- Vous n'avez pas prévu de planification très précise et vous comptez sur les
remarques des enfants, leur spontanéité pour vous adapter à leurs besoins.
- Vous avez prévu une planification assez précise, mais vous adapterez votre
travail selon les opportunités qui s'offriront à vous.

Extrait 6. Ce qui détermine les choix des enseignants pour organiser des rencontres entre les enfants, la vie et le vivant.

Les résultats (figure 3) observés longitudinalement ou transversalement sont cohérents. Les enseignants donnent la priorité à une planification existante, mais souple, de manière à pouvoir s'adapter aux opportunités s'offrant à eux : ces opportunités sont placées au premier rang et absentes des réponses au troisième rang. La priorité est ensuite donnée à une planification très souple s'adaptant aux remarques des enfants. Les enseignants de maternelle rejettent fortement une planification précise sur l'année des rencontres avec la vie et le vivant. L'assertion est placée en troisième position dans les réponses sur la première priorité et en première position pour la troisième priorité (qui est le rejet).

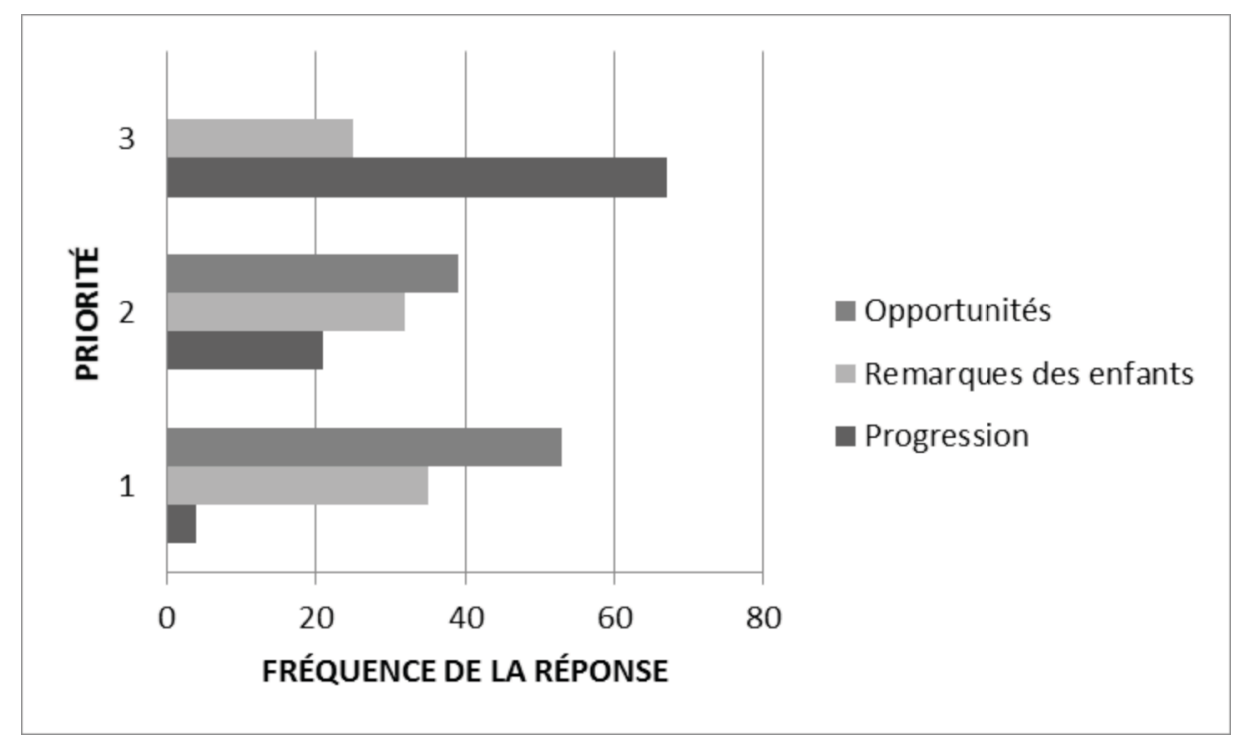

Figure 3. Répartition des choix des questionnés à propos de ce qui pilote leurs choix de l'organisation des rencontres des enfants avec le monde du vivant.

La priorité accordée aux enfants et aux opportunités dans l'organisation des rencontres avec le vivant détermine le caractère improvisé des pratiques enseignantes. Parler d'improvisation n'est pas 
ici pris avec une connotation péjorative. Le terme est considéré dans son acception issue du monde artistique dans lequel l'improvisation est une pratique de créativité spontanée, sur le moment, en réponse au stimulus de l'environnement qui fait appel à des grilles déjà présentes et par la suite développées. C'est à partir de ces grilles que l'artiste va pouvoir développer son imagination. Improviser n'est donc pas quelque chose de facile, c'est ce que font ces enseignants de maternelle, et cela nécessite au contraire des compétences spéciales de créativité et d'inventivité. Ce besoin des enseignants d'être compétents pour improviser est également avancé par Le Boterf (1998) 26 considère que «l'improvisation s'organise autour d'un fil directeur et nécessite la maitrise des techniques de base, elle n'est pas cacophonie ». La recherche restituée montre que ces pratiques enseignantes, organisant des rencontres entre les enfants, la vie et le vivant, se caractérisent par cette improvisation qui renvoie à une compétence déjà mise en avant par Philippe Perrenoud (1994) 27. Les enseignants de maternelles montrent des compétences à gérer l'incertitude et à improviser de manière réglée en classe.

Ce savant mélange pédagogique montre cependant des limites au plan épistémologique: l'improvisation a ses limites, comme le montre l'entretien réalisé avec France (extrait 7).

Comme je t'avais dit, je pensais bien faire un élevage pendant ces périodes 3 et 4, mais l'animateur-science a appelé un jour à l'école pour dire qu'il avait des œufs d'insectes. Alors je me suis lancée un peu au hasard! Il y avait quatre sortes d'œufs au début. Je ne savais pas vraiment de quoi il s'agissait en vrai.

Extrait 7. Deuxième entretien avec France, enseignante en grande section.

France, suivie par carnet de bord, avait précisé qu'elle souhaitait mettre en place un élevage lors du premier entretien, sans savoir quel animal elle choisirait. C'est le hasard qui a déterminé son choix, un appel téléphonique d'un animateur-science de circonscription proposant de donner divers œufs d'insectes. France va donc élever les œufs de quatre espèces différentes sans savoir ce qu'il en sortira, dans combien de temps. Au bout de six semaines, le temps d'une «période», seuls les œufs des phasmes vont éclore et seront observés par les enfants. Les trois autres types d'œufs n'écloront pas lors de l'année scolaire.

Le passage de la transcription d'un entretien (extrait 8) avec l'enseignante Aurélie dont l'extrait 2 avait montré ce qu'elle visait lors de son travail sur l'élevage de têtards.

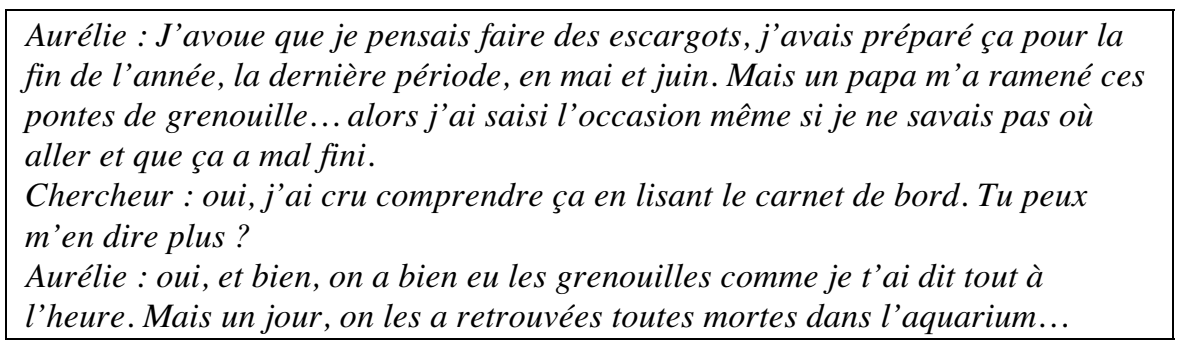

Extrait 8. Troisième entretien avec Aurélie, enseignante en grande section.

Aurélie, enseignante dont la pratique est marquée par des compétences solides à organiser les rencontres entre les enfants, la vie et le vivant, manque cependant de recul épistémologique par rapport au contenu pris en charge. En improvisant un élevage et saisissant une opportunité, elle ne questionne pas le fait qu'élever et «travailler avec» des escargots n'est pas élever des têtards, tant dans les aspects scientifiques, méthodologiques que comportementaux. La mort de l'ensemble des têtards au mois de mai soulève la question du respect de la vie et du rapport au vivant construit alors entre les enfants et les animaux élevés. 
Les données permettent de déceler des faiblesses dans les pratiques, et questionnent fondamentalement la formation des enseignants affectés en école maternelle. Ces enseignants seront en effet amenés à organiser des rencontres entre les enfants et le vivant, et plus largement à prendre en charge les premiers contenus de l'éducation scientifique et technologique. Cette problématique de formation a été ouverte par Coquidé (2007) 28, qui met en avant des éléments spécifiques de la professionnalité à prendre en compte pour penser une formation adaptée pour l'enseignement relative au monde du vivant, des objets et de la matière. Ces éléments spécifiques pris en compte, nous avons formulé un ensemble de propositions pour une formation spécifique des enseignants de l'école maternelle (Charles, 2013) 23. Ces suggestions, fondées sur le curriculum d'éducation scientifique et technologique, peuvent être également étendues à d'autres domaines d'enseignement-apprentissages, afin de concourir à la formation des enseignants de l'école avant six ans : tant de choses se jouent en maternelle qu'il est nécessaire de former des professionnels de l'enseignement et des apprentissages de la petite enfance.

\section{Références}

1. F. Charles, Découvrir le monde de la nature et des objets avant six ans à l'école maternelle: spécificités du curriculum, spécialité des enseignants, Thèse de Doctorat de l'Université Paris Descartes (2012).

2. J. Bisault, Contribution à l'élaboration curriculaire d'une éducation scientifique à l'école maternelle : modélisation des moments scolaires à visée scientifique, Mémoire d'Habilitation à Diriger des Recherches, École Normale Supérieure de Cachan (2011).

3. J. Lebeaume, L'éducation technologique, histoire et méthodes (Paris, ESF, 2000).

4. A. Prost, Histoire générale de l'enseignement et de l'éducation en France, tome 4 (Paris, Nouvelle Librairie de France, 1981).

5. É. Plaisance, L'enfant, la maternelle, la société (Paris, Presses Universitaires de France, 1986).

6. Ministère de l'Éducation Nationale, Bulletin Officiel spécial du 26 mars 2015 (2015).

7. Ministère de l'Éducation Nationale, Bulletin Officiel hors-série du 20 février 2008 (2008).

8. C. Orange, É. Plé, Les sciences de 2 à 10 ans, l'entrée dans la culture scientifique, Aster 31, 1-8 (2000).

9. M. Coquidé, J. Lebeaume, La découverte de la nature et des objets à l'école, hier et aujourd'hui, Grand N, 72, 105-114 (2003).

10. M. Coquidé, Les pratiques expérimentales : propos d'enseignants et conceptions officielles, Aster, 26, 109-132 (1998).

11.C. Lasson, Ruptures et continuités dans la familiarisation pratique en technologie de l'école préélémentaire au collège, Thèse de Doctorat de l'École Normale Supérieure de Cachan (2004).

12. C. Ledrapier, Le rôle de l'action dans l'éducation scientifique à l'école maternelle: cas de l'approche des phénomènes physiques, Thèse de Doctorat de l'École Normale Supérieure de Cachan (2007).

13. J. Bisault, Moments d'éducation scientifique à l'école maternelle et rencontre avec des objets du quotidien, Skholê, 18(2), 9-18 (2014).

14. M. Coquidé, Discours d'enseignants de maternelle sur des espaces pour découvrir le monde, Skholê, 18(2), 31-40 (2014).

15.F. Charles, Compositions et tissage dans les pratiques de prise en charge du curriculum d'éducation scientifique et technologique à l'école maternelle, Skholê, 18(2), 19-30 (2014).

16. A. Florin et $\mathrm{C}$. Crammer, Enseigner à l'école maternelle, de la recherche aux gestes professionnels (Paris, Hatier, 2009).

17.F. Cros, L'écriture sur la pratique est-elle un outil de professionnalisation?, Perspectives documentaires en éducation, 58, 41-47 (2003).

18. I. Vinatier, Pour une didactique professionnelle de l'enseignement (Rennes, Presses Universitaires de Rennes, 2009). 
19.G. Baillat, Rapport de recherche, Enquête sur la polyvalence des enseignants du premier degré (Reims, IUFM de Reims Champagne-Ardenne, 2001).

20. G. Baillat, Rapport de recherche, Polyvalence, conceptions didactiques et partage du travail chez les enseignants du premier degré (Reims, IUFM de Reims Champagne-Ardenne, 2003).

21.J. Guichard, Observer pour comprendre les sciences de la vie et de la Terre (Paris, Hachette Éducation, 1998).

22. M. Develay, De l'apprentissage à l'enseignement (Paris, ESF,1992).

23. F. Charles, Des moments d'éducation scientifique et technologique à l'école maternelle (19702008) ; contenus, entremêlements et enracinement historique des pratiques contemporaines, Grand $N$, 92, 67-96 (2013).

24. É. Prairat, Normes et devoirs professionnels, l'esprit déontologique, Les sciences de l'éducationPour l'Ère nouvelle, 45, 1-2, 76-91 (2012).

25. D. Bucheton et Y. Soulé, Les gestes professionnels et le jeu des postures de l'enseignant dans la classe : un multi-agenda de préoccupations enchâssées, Éducation \& Didactique, 3(3) 29-48 (2009).

26. G. Le Boterf, Évaluer les compétences. Quels jugements? Quels critères ? Quelles instances ?, Éducation permanente, 135, 143-152 (1998).

27. P. Perrenoud, La formation des enseignants entre théorie et pratique (Paris, L'Harmattan, 1994).

28. M. Coquidé, Quels contenus de formation pour enseigner à l'école maternelle ? L'exemple de la formation à l'activité « faire découvrir la nature et les objets », Recherche et formation, 55, 75-92 (2007). 
